\title{
Técnicas de manejo em Odontopediatria: uma revisão narrativa da literatura
}

\author{
Management techniques in Dental Pediatrics: a narrative review of the literature \\ Técnicas de manejo en Pediatría Dental: una revisión narrativa de la literatura
}

Recebido: 16/08/2021 | Revisado: 22/08/2021 | Aceito: 02/09/2021 | Publicado: 05/09/2021

\author{
Victor Felipe Davino Coelho \\ ORCID: https://orcid.org/0000-0002-3396-2105 \\ Centro Universitário Mario Pontes Jucá, Brasil \\ E-mail: victorfelipedavinocoelho@ hotmail.com \\ Lucas Vinicius Davino Coelho \\ ORCID: https://orcid.org/0000-0002-9747-0855 \\ Centro Universitário Mario Pontes Jucá, Brasil \\ E-mail: lucasdavino20@gmail.com \\ Ana Maria Guerra Costa \\ ORCID: https://orcid.org/0000-0002-6564-4718 \\ Centro Universitário Mario Pontes Jucá, Brasil \\ E-mail: anaguerracosta@yahoo.com.br
}

\begin{abstract}
Resumo
Introdução: o presente estudo objetiva a explanação descritiva das técnicas de manejo em odontopediatria, que visam desenvolver na criança um comportamento mais apropriado enquanto recebe o tratamento odontológico. Tal conduta é extremamente necessária para que o profissional consiga realizar o tratamento de forma adequada e eficiente, sem causar danos à criança. Metodologia: foram utilizados os descritores em ciência da saúde (DeCS/MESH) para a elaboração da estratégia de busca, a qual foi aplicada nas bases de dados PUBMED, SCOPUS e WEB OF SCIENCE, sendo encontrados ao todo 231 artigos. Os critérios de inclusão dos artigos foram: abordar no título a temática do cuidado com a criança no contexto da odontopediatria e trazer a descrição de alguma técnica de manejo com crianças durante a realização de procedimentos odontológicos utilizada por dentistas e assistentes. Como critérios de exclusão foram excluídos quaisquer trabalhos que tratassem sobre aspectos puramente teóricos, adolescentes ou que relatassem técnicas de sedação. Após a retirada das duplicatas e aplicação dos critérios inclusão e exclusão, foram analisados 9 artigos. Resultados e discussões: foram descritas 8 técnicas de manejo de comportamento, contudo, existe a necessidade de habilidade técnica e conhecimento teórico para a escolha e emprego do manejo correto. Além disso, para se trabalhar com crianças e obter a colaboração da mesma durante os procedimentos odontológicos, é necessário inicialmente conhecer e respeitar cada fase do desenvolvimento da criança. Conclusão: o profissional deve combater o medo da criança tornando o ambiente do consultório em algo familiar, utilizando as técnicas de manejo mais adequadas.
\end{abstract}

Palavras-chave: Controle comportamental; Medo; Odontopediatria.

\begin{abstract}
Introduction: this study aims to provide a descriptive explanation of management techniques in pediatric dentistry, which aim to develop a more appropriate behavior in the child while receiving dental treatment. Such conduct is extremely necessary for the professional to be able to carry out the treatment properly and efficiently, without causing harm to the child. Methodology: the descriptors in health science (DeCS / MESH) were used to elaborate the search strategy, which was applied in the PUBMED, SCOPUS and WEB OF SCIENCE databases, being found among all 231 articles. The inclusion criteria for the articles were: addressing in the title the theme of caring for a child in the context of pediatric dentistry and providing a description of some handling techniques with children during the performance of dental procedures used by dentists and assistants. As exclusion criteria, any papers that dealt with purely theoretical aspects, adolescents or that reported sedation techniques were excluded. After removing the duplicates and applying the inclusion and exclusion criteria, 9 articles were allowed. Results and discussions: 8 behavior management techniques were obtained, however, there is a need for technique and theoretical knowledge to choose and use the correct management. Furthermore, to work with children and obtain their cooperation during dental procedures, it is necessary to know and respect each stage of the child's development. Conclusion: the professional must fight the fear of the child by making the office environment something familiar, using the most trained management techniques.
\end{abstract}

Keywords: Behavior control; Fear; Pediatric dentistry. 


\begin{abstract}
Resumen
Introducción: este estudio tiene como objetivo proporcionar una explicación descriptiva de las técnicas de manejo en odontopediatría, que tienen como objetivo desarrollar un comportamiento más adecuado del niño durante el tratamiento odontológico. Esta conducta es sumamente necesaria para que el profesional pueda realizar el tratamiento de manera adecuada y eficaz, sin dañar al niño. Metodología: se utilizaron los descriptores en ciencias de la salud (DeCS / MESH) para desarrollar la estrategia de búsqueda, la cual se aplicó en las bases de datos PUBMED, SCOPUS y WEB OF SCIENCE, con un total de 231 artículos encontrados. Los criterios de inclusión de los artículos fueron: abordar el tema del cuidado infantil en el contexto de la odontopediatría en el título y describir algunas técnicas de manejo con niños durante la realización de procedimientos dentales utilizados por odontólogos y asistentes. Como criterio de exclusión, se excluyeron aquellos trabajos que abordaran aspectos puramente teóricos, adolescentes o que informaran sobre técnicas de sedación.. Tras eliminar los duplicados y aplicar los criterios de inclusión y exclusión, se analizaron 9 artículos. Resultados y discusiones: Se describieron 8 técnicas de manejo del comportamiento, sin embargo, es necesario contar con habilidades técnicas y conocimientos teóricos para elegir y utilizar el manejo correcto. Además, para trabajar con los niños y obtener su cooperación durante los procedimientos dentales, es necesario, inicialmente, conocer y respetar cada etapa del desarrollo infantil. Conclusión: el profesional debe combatir el miedo del niño familiarizando el ambiente de la oficina, utilizando las técnicas de gestión más adecuadas.
\end{abstract}

Palabras clave: Control de la conducta; Temor; Odontólogo pediátrico.

\title{
1. Introdução
}

Devido ao medo dentário e a pouca cooperação por parte das crianças durante os atendimentos odontológicos, fez-se necessário o desenvolvimento e estabelecimento de técnicas de manejo para com esse público (Savanheimo \& Vehkalahti, 2014) Tais técnicas objetivam desenvolver na criança um comportamento mais cooperativo enquanto recebe o tratamento odontológico. Cabe ressaltar que a incorporação dessas técnicas pelo profissional da odontologia é extremamente necessária, uma vez que ela busca tonar os tratamentos clínicos mais efetivos, evitando assim reincidências do problema, bem como auxilia na redução dos índices de erros de conduta e consequente dano ao paciente. Além disso, o manejo infantil ainda visa estabelecer uma comunicação com a criança, educar o paciente, construir uma relação de confiança, além de prevenir e aliviar o medo e a ansiedade (Baghdadi, Jbara \& Muhajarine, 2021).

Ademais, é comum que, desde a infância, os pacientes ou seus responsáveis optem por procurar os serviços odontológicos apenas quando apresentam dor ou alguma outra alteração anatômica na cavidade bucal. Essa cultura contribui para a associação negativa com a figura do dentista e o ambiente odontológico (Pine et al., 2015). Com base nisso, Silva et al. (2016) aponta que a forma mais adequada de abordagem do público infantil está condicionada à desconstrução dessa associação negativa, por meio do estímulo ao comportamento apropriado mediante recompensas. Desse modo, o ambiente clinico deve ser agradável, com demonstrações físicas da equipe odontológica de afeição e cuidado com a criança, elogiando-a, permitindo os abraços, disponibilizando lembrancinhas, algo que a felicite pelo seu bom comportamento, proporcionando à criança uma experiência positiva (Simões et al.,2016; Gao, Hamzah, Yiu, Mcgrath \& King, 2013).

Outro ponto importante a ser abordado é prática da promoção do cuidado dentário, pois, uma vez que os pacientes comecem a associar, desde de cedo, a figura do cirurgião-dentista aos conceitos de saúde, bem estar, prevenção de doenças e autocuidado, certamente haverá uma maior procura desses profissionais. Isso permitirá a prevenção de agravos que demandam procedimentos odontológicos mais dolorosos e traumatizantes (Vigu \& Stanciu, 2019). Dessa forma, destaca-se a necessidade de que os cirurgiões dentistas assumam o papel de promotores de saúde, na medida que atuem evitando o desenvolvimento de doenças e não apenas tratando-as (Aljafari, Rice, Elizabeth Gallagher \& Therese Hosey, 2015).

Além disso, é importante que os próprios pais sejam instruídos a transmitirem aos seus filhos uma imagem positiva sobre a ida ao consultório odontológico, explicando que a visita ao dentista é importante para evitar problemas de saúde, de modo que quando mais frequentes forem as visitas, menos processos dolorosos haverão visto que os dentes ficarão mais saldáveis (Kaur et al., 2019).

No mais, para se estabelecer um vínculo entre a criança e o dentista é necessário que ele faça o uso de estratégias de 
comunicação mais direcionadas, tais como a modulação de voz, expressão facial, frases verbais e demonstrações físicas de afeição. Por sua vez, os gestos positivos como expressão facial tem como objetivo recompensar comportamentos desejados, facilitando o processo do atendimento (Ferreira et al., 2016; Yuan, Humphris, Macpherson, Ross \& Freeman, 2021).

Contudo, as situações mais difíceis enfrentadas pelos odontopediatras são aquelas em que as crianças, principalmente as mais novas, não colaboram com a realização do tratamento, chorando, gritando e realizando movimentando na tentativa de tentativas de sair da cadeira odontológica. Deve-se compreender, no entanto, que esses comportamentos não-colaborativos são, geralmente, atribuídos ao medo, a traumas ou a outros fatores inerentes ao indivíduo (Eleutério, Oliveira \& Júnior, 2011; Bettega et al., 2012). Portanto, nesses contextos torna-se ainda mais importante a incorporação de técnicas de que auxiliem a equipe odontológica no manejo com crianças pouco colaborativas (Aljafari, Rice, Gallagher \& Hosey, 2015; Silva, Anabuki, Viana, Corrêa-Faria, Moterane, Tedesco, Costa, Hosey \& Raggio, 2021).

Frente ao exposto, a presente revisão de literatura objetiva catalogar as técnicas de manejo com crianças no contexto da odontopediatria, de modo a auxiliar os profissionais da odontologia a proporcionarem um ambiente mais favorável à prática clínica adequada e garantir que as crianças vivenciem uma experiência de cuidado odontológico positiva.

\section{Metodologia}

Com base na metodologia de Honório e Santiago (2021), para a realização desta revisão narrativa de literatura foram utilizados os descritores em ciência da saúde (DeCS/MESH) e seus respectivos termos entry. Por sua vez, foi elaborada uma estratégia de busca, Child AND "Child Behavior" AND "Health Behavior" AND "Dental Care", com base no sistema PICo (população, incidência e contexto) utilizando-se o operador booleano AND entre os descritores. Tal estratégia foi utilizada para a busca de artigos científicos em todos os idiomas e sem limite da data de publicação nas bases de dados PUBMED, SCOPUS e WEB OF SCIENCE, sendo encontrados ao todo 231 artigos. Os critérios de inclusão dos artigos foram: abordar no título a temática do cuidado com a criança no contexto da odontopediatria e trazer a descrição de alguma técnica de manejo com crianças durante a realização de procedimentos odontológicos utilizada por dentistas e assistentes. Como critérios de exclusão foram excluídos quaisquer trabalhos que tratassem sobre aspectos puramente teóricos, adolescentes ou que relatassem técnicas de sedação. Após a retirada das duplicatas e aplicação dos critérios inclusão e exclusão, foram lidos e analisados na íntegra 9 artigos.

\section{Resultados}

Foram encontradas na literatura oito técnicas de manejo com crianças no contexto da odontopediatria.

Técnica 1: Falar-Mostrar-Fazer: "O Dentista primeiro mostra para a criança o que será usado em sua boca, depois explica como será usado. Então, demonstra, primeiro na mão da criança e depois em sua boca, o que será feito”.

Técnica 2: Controle da Voz: "Conquistamos a atenção da criança mudando o tom ou aumentando o volume da voz. A maneira que falamos é mais importante que as palavras que usamos".

Técnica 3: Reforço Positivo: "Cada vez que a criança se comporta bem, o dentista faz um elogio ou entrega um presente, mostrando que gostou daquele comportamento".

Técnica 4: Modelo: "A criança assiste a uma demonstração de um atendimento odontológico através de uma fita de vídeo, teatrinho ou assistindo ao atendimento de uma outra pessoa".

Técnica 5: Mão-sobre-a-boca: “A assistente do dentista segura a criança que está fazendo birra, enquanto o dentista coloca a sua mão sobre a boca da criança e fala em tom suave, perto do ouvido da criança, que ela pare de chorar e gritar e escute".

Técnica 6: Contenção Ativa: “A assistente do Dentista segura os braços e as pernas da criança, evitando que ela se 
movimente enquanto o dentista trabalha".

Técnica 7: Contenção Passiva: "O dentista utiliza tecidos ou faixas de contenções apropriados para enrolar a criança, evitando que ela se movimente durante $\mathrm{o}$ atendimento".

Técnica 8: Sedação: "A criança toma remédios indicados pelo dentista e fica sonolenta durante o atendimento".

Segundo Silva et al. (2016) a distração é um método que busca desviar a atenção do paciente, utilizando música, filmes, livros ou histórias, sendo bastante eficaz no processo no tratamento odontológico infantil.

O envolvimento do paciente em comandos verbais, com frases adequadas, seguida uma demonstração visual e tátil, buscando tranquilizar o paciente infantil no procedimento é bastante utilizada Silva et al. (2016).

A comunicação verbal é uma ferramenta eficaz, deve ser uma fonte única para a criança durante o procedimento, deve ser evitado que o ambiente tenha várias pessoas falando ao mesmo tempo, evitando que a criança fique confusa. (Albuquerque et al., 2010)

Lamha e Costa (2012) empregaram a mesma técnica verificando que o atendimento da criança quando associado com a utilização do brinquedo, faz com que a mesma se sinta confiante e aceite com maior facilidade o atendimento odontológico.

Para Albuquerque et al. (2010) as técnicas de manejo comportamental podem ser amplamente utilizadas na abordagem da criança no consultório odontológico, possibilitando, assim, a otimização do atendimento. Entretanto, para o sucesso do atendimento.

Nesse contexto Ferreira J (2009) ressalta que o atendimento odontológico infantil deve obter um gerenciamento comportamental da criança, de forma a viabilizar exames e intervenções relacionados à promoção de saúde. A abordagem deve se adequar à idade, ao gênero, ao estado de saúde geral e aos fatores familiares da criança.

\section{Discussão}

Simões et al. (2016), relata que de acordo com a idade, gênero, nível socioeconômico, estado de saúde geral e bucal e fatores familiares, deve ser escolhida a técnica adequada promovendo assim o gerenciamento do comportamento de forma a possibilitar as intervenções. Para isso, o conhecimento e habilidades no manejo dos procedimentos, se faz necessário. Os achados de Shitsuka et al. (2019) corroboram com os fatores familiares, elencando que a ansiedade dos pais pode ser fator determinante para as dificuldades de tratamento pediátrico.

Singh et al. (2014), cita a importância das técnicas de comportamento utilizadas na odontopediatria que tem como principal objetivo manter a atenção e cooperação do paciente. Através de técnicas básicas para adaptação de comportamento, a criança pode ser controlada de forma eficaz.

Nesse contexto, Albuquerque (2010) assevera a recepção da criança no consultório, a conversa durante a sua transferência da sala e durante o preparo da criança na cadeira odontológica. Ter cuidado na comunicação como regra, um papel mais passivo, porque a criança só ouve uma pessoa de cada vez. O relato de Santos et al (2020) reforçam a importância do acolhimento desde o momento da sala de recepção, reforçando que a preocupação com o manejo infantil deve estar planejado de forma integral.

Para Silva et al. (2016), a transmissão das mensagens para a criança deve ser sempre na primeira pessoa, para não haver uma avaliação negativa da criança e evitar problema na condução dos procedimentos odontológicos. $\mathrm{O}$ autor ainda relata que a aplicação de procedimentos eficientes para a distração da criança é um fator importante para aliviar a tensão psicológica gerada pela situação dentro do consultório que pode acarretar ansiedade e medo no paciente. Desta forma, tornar o ambiente confortável ao tratamento, deve ser um dever para o dentista, podendo alcançar assim um melhor resultado. Algumas estratégias de manejo podem ser utilizadas como músicas, vídeos e histórias infantis.

Elogiar verbalmente o paciente tem um resultado bem positivo, a forma amável de falar da aprovação do 
comportamento trás segurança a criança. Outra técnica usada é a utilização de brindes como, crachás, brinquedos, adesivos, dentre outros, como forma de recompensar o bom comportamento do paciente. (Singh et al., 2014; Simões et al., 2016)

Para Singh et al. (2014) a utilização de ferramentas como desenhos animados, livros, música ou histórias, falar com os pacientes enquanto trabalham, tira a atenção do paciente para longe do procedimento odontológico, diminuindo a ansiedade gerada no atendimento.

Desta forma Silva et. al. (2016), destaca a técnica da "mão sobre a boca", que tem como sua característica a firmeza na condução de colocar a criança na cadeira odontológica. Se a criança movimentar braços e pernas, o dentista e a auxiliar conterão a criança, prevenindo seu próprio dano e danos à equipe e ao equipamento. O profissional posiciona suas mãos sobre a boca do paciente infantil, com o objetivo de abafar qualquer som e simultaneamente promover a aproximação no ouvido da criança.

Albuquerque et al. (2010) relaciona o medo da anestesia como fator principal para reações negativas do paciente. Desta forma, entende-se que esse sentimento pode ser evitado quando as crianças são levadas ao dentista desde bebês. Deste modo, o consultório se torna algo familiar, ambiente que faz parte da vida delas. O medo deve ser um fator extremamente considerado, entretanto, como demonstrado pelo estudo piloto de Andrade et al (2020), tal fator, apesar de presente, não é, ao contrário do senso comum, uma constante na população infantil. Na verdade, os autores demonstraram que uma correta apresentação e acolhimento pode ofertar conforto e segurança nas primeiras experiências odontológicas e controlar esse fator de maneira eficaz.

Nesse contexto, o Odontopediatra deve estar capacitado na condução dos procedimentos terapêuticos e por se tratar de atendimento a crianças, os pais devem estar de comum acordo, participar ativamente das tomadas de decisões e, principalmente, estarem esclarecidos quanto aos métodos de controle comportamental. (Simões, 2016)

Segundo Vieira (2017), a técnica proposta aplicada, pode não surtir o efeito desejado em casos de criança sem o discernimento suficiente para executar o proposto. Assim, o profissional deve ter o discernimento sobre qual técnica deverá ser aplicada de acordo com a necessidade e estágio de cada criança, sendo a mais utilizada inicialmente, a técnica onde se diz e mostra como será feito o procedimento odontológico.

De forma conclusiva, Boro (2016), entende que a criança deve conhecer as funções dos equipamentos e procedimentos a serem realizados, familiarizando e diminuindo assim a reação negativa ao tratamento proposto pelo profissional.

Mostrar ao paciente o que será realizado no procedimento odontológico com comando de verbal e não verbal firme é uma ferramenta de grande importância ao atendimento odontológico, deixando o paciente confortável em relação ao procedimento, e assim adaptando-o para se obter respostas positivas para o atendimento. (Albuquerque et al. ,2010)

Para Albuquerque et al. (2010) a postura que o dentista deve adotar em um atendimento em odontopediatria de maneira passiva, afim de que o auxiliar possa passar ao paciente as regras durante o preparo da criança para o procedimento odontológico.

Deve ser observado o comportamento da criança no atendimento clinico para a execução de estratégias comportamentais em Odontopediatria e melhorar o comportamento da criança. (Boro, 2016).

A relação entre o profissional o paciente e o responsável pela criança, facilita na abordagem necessária para a realização dos procedimentos e o manejo do comportamento (Albuquerque et. al., 2010).

Segundo Albuquerque et. al. (2010) pacientes pediátricos tem reações adversas em situações na qual não compreende, choro, a recusa em abrir a boca, resistência se até mesmo o vômito são comportamentos comuns.

A comunicação não verbalizada, esta adicionada na acolhida do paciente, o contato expressão facial e a linguagem corporal para orientar o comportamento do paciente são ferramentas base para qualquer atendimento indicado para ser usada 
em qualquer idade. (Silva et al. ,2016)

Singh et al, (2014) relata que alguns dentistas na tentativa de obter o controle do ambiente muda o tom da voz, com um tom forte, ameaçador, originando um comportamento negativo na criança. Entender a técnica e executar com suavidade tranquila para que a criança não se assuste facilitando o atendimento.

\section{Considerações Finais}

Após a revisão de literatura, destacamos que para se trabalhar com crianças e obter a colaboração das mesmas, durante os procedimentos odontológicos, é necessário, inicialmente, conhecer e respeitar cada fase do desenvolvimento da criança. A partir daí, escolher e empregar corretamente a técnica de controle comportamental mais adequada a cada situação. O profissional deve entender que cada técnica deverá ser aplicada de acordo com a necessidade de cada paciente, sendo a mais utilizada o "controle pela voz" e "dizer-mostrar-fazer", e a mais controversa é a da "mão sobre a boca".

Desta forma, o profissional deve combater o medo da criança tornando o ambiente do consultório transformando-o em algo familiar, ambiente que faz parte da vida delas. Nesse contexto, o dentista deve entender o estágio de desenvolvimento da criança para aplicação correta do manejo, de forma que a colaboração dure durante os procedimentos odontológicos e para que isto ocorra, deve-se respeitar a fase da criança.

\section{Referências}

Albuquerque, C. M., Gouvêa, C. V. D., Moraes, R. C. M., Barros, R. N. \& Couto, C. F. (2010). Principais Técnicas De Controle de comportamento em Odontopediatria. Arquivos em odontologia, 45 (2).

Aljafari, A., Rice, C., Elizabeth Gallagher, J. \& Therese Hosey, M. (2015). An oral health education video game for high caries risk children: study protocol for a randomized controlled trial. Trials. 16 (237).: 10.1186/s13063-015-0754-6.

Andrade, N. M., Laureano, I. C. C., Farias, L., Fernandes, L. H. F., \& Cavalcanti, A. L. (2020). Medo odontológico em escolares: um estudo piloto utilizando o Children's Fear Survey Schedule - Dental Subscale. Research, Society and Development, 9(5), e26953124. https://doi.org/10.33448/rsd-v9i5.3124

Baghdadi, Z. D., Jbara, S. \& Muhajarine, N. (2021). Children and Parents Perspectives On Children's Dental Treatment Under General Anesthesia: A Narratology From Saskatoon, Canada. European Archives Of Paediatric Dentistry, 22 (4), 725-737, dx.doi.org/10.1007/s40368-021-00613-6.

Carvalho, A. A. (2011). Percepção De Mães Sobre Técnicas De Condução Do Comportamento De Crianças Em Atendimento Odontológico E Sua Randomização. Dissertação (Mestrado Em Ciências Da Saúde) - Universidade Federal De Goiás, Goiânia-Go.

Ferreira, J., Aragão, A. \& Colares, V. (2009). Técnicas de Controle do Comportamento do Paciente Infantil: Revisão De Literatura. Pesquibras Odontopediatria Clín, 247-251.

Gao, X., Hamzah, S., Yiu, C. K. Y., Mcgrath, C. \& King, N. M. (2013). Dental Fear And Anxiety In Children And Adolescents: Qualitative Study Using Youtube. Journal of Medical Internet Research, 15, (2), Dx.Doi.Org/10.2196/Jmir.2290.

Honório, H. M. \& Santiago Júnior, J. F. (2021). Fundamentos das Revisões Sistemáticas em Saúde. Santos Publicações.

Kaur, S., Lingesha Telgi, R., Tandon, V., Kaur, R. \& Bhattacharyya, S. (2019). Parents outlook on preventive dental modalities for their children in Udham Singh Nagar, India. J Educ Health Promot (8).

Oliveira, W. F., Fonseca, C. N., Bruzinga, F. F. B., Souza, A. F., Oliveira, L. F. B., Oliveira, C. C. (2012). Técnicas alternativas para o atendimento em odontopediatria: uma revisão aplicada à clínica. EFDeportes.com, revista digital (182).

Santos, L. G. P., Rodrigues, D. C., Mariz, W. S., Torres, J. L. M., Pinheiro, N. S. de S. L., Macena, M. C. B., Rodrigues, R. de Q. F., \& Fonseca, F. R. A. (2020). Manejo do comportamento infantil e saúde bucal na sala de espera: um relato de experiência. Research, Society and Development, 9(7), e888975069. https://doi.org/10.33448/rsd-v9i7.5069

Savanheimo, N. \& M Vehkalahti, M., (2014). Acompanhamento de cinco anos de crianças recebendo atendimento odontológico abrangente sob anestesia geral. BMC Oral Health, 20 (2).

Sharma, A., Kumar, D., Anand, A., Mittal, V., Singh, A., \& Aggarwal, N. (2017). Factorspredictingbehaviour Management Problemsduringinitial Dental Examination In Childrenaged 2 To 8 Years. Int J Clinpediatrdent, 10 (1).

Shitsuka, C., Friggi, M. N. P., \& Volpini, R. M. C. (2019). Influência dos pais sobre o comportamento infantil no atendimento odontológico. Research, Society and Development, 8(7), e43871154. https://doi.org/10.33448/rsd-v8i7.1154 
Research, Society and Development, v. 10, n.11, e414101119489, 2021

(CC BY 4.0) | ISSN 2525-3409 | DOI: http://dx.doi.org/10.33448/rsd-v10i11.19489

Silva, G. S. da, Anabuki, A. A. Viana, K. A, Corrêa-Faria, P, Moterane, M. M., Tedesco, T. K., Costa, P. S., Hosey, M. T., \& Raggio, D. P. (2021). Sedation Versus Protective Stabilization For Dental Treatment Of Children With Caries And Challenging Behavior At The Dentist (Choose): A Study Protocol For A Non-Randomized Clinical Trial. Bmc Oral Health, 21 (1), 1-.2. Dx.Doi.Org/10.1186/S12903-021-01594-0.

Silva, L. F. P., Freire, N. C., Santana, R. S. \& Miasato, J. M. Técnicas De Manejo Comportamentais não farmacológicas na odontopediatria. Rev. Odontol. $135-42$.

Simões, F. X. C. P., Macedo, T. G., Coqueiro, R. S. \& Pithonm (2016). Percepção Dos Pais Sobre As Técnicas De Manejo Comportamental Utilizadas Em Odontopediatria. Rev. Bras. Odontol., 73(4), 277-82.

Singh, H., Rehman, R., Kadtane., Dalai, D. R. \& Jain, C. D. (2014). Techniques For The Behavior Management In Pediatric Dentistry, International Journal Of Scientific Study, India, 2.

Vieira, L. D. S., Bezerra, R. N., Varella, P. L. S., Peixoto, M. L. B. \& Oliveira, M.S. (2017). Manejo Comportamental na clínica de odontopediatria. XVII Safety, Health and Environment World Congress. 10.14684/SHEWC.17.2017.84-85.

Vigu, A. \& Stanciu, D. (2019). When the fear of dentist is relevant for more than one's oral health. A structural equation model of dental fear, self-esteem, oral-health-related wellbeing, and general well-being. Dove Press jornal. 